LAWRENCE LIVERMORE N A T IO N A L LABORATORY
Simple Derivation of the Hauser-Feshbach and Weisskopf-Ewing Formulae, with Application to Surrogate Reactions

Frank S. Dietrich

January 7, 2004 
This document was prepared as an account of work sponsored by an agency of the United States Government. Neither the United States Government nor the University of California nor any of their employees, makes any warranty, express or implied, or assumes any legal liability or responsibility for the accuracy, completeness, or usefulness of any information, apparatus, product, or process disclosed, or represents that its use would not infringe privately owned rights. Reference herein to any specific commercial product, process, or service by trade name, trademark, manufacturer, or otherwise, does not necessarily constitute or imply its endorsement, recommendation, or favoring by the United States Government or the University of California. The views and opinions of authors expressed herein do not necessarily state or reflect those of the United States Government or the University of California, and shall not be used for advertising or product endorsement purposes.

This work was performed under the auspices of the U.S. Department of Energy by University of California, Lawrence Livermore National Laboratory under Contract W-7405-Eng-48. 


\title{
Simple Derivation of the Hauser-Feshbach and Weisskopf-Ewing Formulae, with Application to Surrogate Reactions
}

\author{
Frank S. Dietrich
}

January 3, 2004

\section{Introduction}

The analysis of surrogate reactions, an indirect technique for determining cross sections on unstable nuclei, relies heavily on compound-nuclear reaction models. The purpose of this tutorial is to provide an introduction to the principal models, based on the Hauser-Feshbach and Weisskopf-Ewing formalisms, and to show how they are applied to surrogate reactions. The outline of the tutorial is the following:

- Section 2 gives a simple derivation of the Hauser-Feshbach formula based on an energy average over well-separated Breit-Wigner resonances. This derivation is strictly correct only at low energies where the resonances do not overlap; i.e.

$$
<\Gamma>/ D \ll 1
$$

where $\langle\Gamma\rangle$ is the average total width of the resonances of a given spin $J$, and $D$ is the average spacing of these resonances. The resultant expression shows under what circumstances the compound nuclear assumption (independence of formation and decay) is valid. More complicated derivations of statistical reaction theory for cases where resonances overlap are available (e.g. Kawai, Kerman, and McVoy (KKM)).

- Section 3 discusses the conditions in which the Hauser-Feshbach formula, which rigorously conserves total angular momentum and parity, reduces to the simpler and earlier Weisskopf-Ewing result in which these quantities are not conserved.

- Section 4 shows how the Hauser-Feshbach and Weisskopf-Ewing formulas are applied in making indirect measurements of cross sections by the surrogate technique. A procedure for modifying existing Hauser-Feshbach codes so that they are suitable for analysis of surrogate reactions is indicated. 


\section{Derivation of the Hauser-Feshbach formula}

We begin with the expression for a reaction from channel $a$ to channel $b$ proceeding through well-separated compound nuclear resonances of spin $J$, expressed as a sum over Breit-Wigner resonances:

$$
\sigma_{a b}^{J}(E)=\pi \lambda^{2} \omega_{a}^{J} \sum_{\lambda} \frac{\Gamma_{\lambda a} \Gamma_{\lambda b}}{\left(E-E_{\lambda}\right)^{2}+\frac{1}{4} \Gamma_{\lambda}^{2}}
$$

where $E$ is the incident energy, $\lambda$ is the reduced wavelength in the incident channel (inverse of the wave number $k), \omega_{a}^{J}$ is a statistical factor, $\Gamma_{\lambda a}$ and $\Gamma_{\lambda b}$ are the partial widths of resonance $\lambda$ in the incident and exit channels, $E_{\lambda}$ is the energy of the resonance and $\Gamma_{\lambda}$ is its total width. The statistical factor is

$$
\omega_{a}^{J}=\frac{2 J+1}{\left(2 i_{a}+1\right)\left(2 I_{a}+1\right)}
$$

where $i_{a}$ and $I_{a}$ are the projectile and target spins; for incident photons the factor $\left(2 i_{a}+1\right)$ is replaced by 2 . The total width is the sum of the partial widths in all channels,

$$
\Gamma_{\lambda}=\sum_{c} \Gamma_{\lambda c}
$$

In writing the cross section as a sum over resonances we have ignored a smooth background amplitude and its interference with the resonances. This background is associated with direct reactions and is not of interest here. We have also suppressed explicit reference to the parity.

Since Hauser-Feshbach is a theory for the energy-averaged cross section, we now average Eq. 2 over an energy interval $\Delta$ large enough to contain many resonances; that is, $D / \Delta \ll 1$, where $D$ is the average spacing of resonances of spin $J$. To do this, we note that the energy integral over a single term in the sum in Eq. 2 is

$$
\int_{0}^{\infty} d E \frac{\Gamma_{\lambda a} \Gamma_{\lambda b}}{\left(E-E_{\lambda}\right)^{2}+\frac{1}{4} \Gamma_{\lambda}^{2}}=2 \pi \frac{\Gamma_{\lambda a} \Gamma_{\lambda b}}{\Gamma_{\lambda}} .
$$

Taking the energy average of Eq. 2 then yields

$$
\left\langle\sigma_{a b}^{J}(E)\right\rangle=\pi \lambda^{2} \omega_{a}^{J} \frac{2 \pi}{D}\left\langle\frac{\Gamma_{a} \Gamma_{b}}{\Gamma}\right\rangle .
$$

Note that this expression does not contain the energy averages of the individual widths, but rather the average of the combination $\Gamma_{a} \Gamma_{b} / \Gamma$. This distinction is important because in general there are correlations between the widths. Accordingly, we define a width fluctuation factor $W_{a b}$ by

$$
\left\langle\frac{\Gamma_{a} \Gamma_{b}}{\Gamma}\right\rangle=W_{a b} \frac{\left\langle\Gamma_{a}\right\rangle\left\langle\Gamma_{b}\right\rangle}{\langle\Gamma\rangle}
$$

so that the energy-averaged cross section may be expressed as

$$
\left\langle\sigma_{a b}^{J}(E)\right\rangle=\pi \lambda^{2} \omega_{a}^{J} \frac{2 \pi}{D} \frac{\left\langle\Gamma_{a}\right\rangle\left\langle\Gamma_{b}\right\rangle}{\langle\Gamma\rangle} W_{a b}
$$


In the limit $\left\langle\Gamma_{c}>/ D \ll 1\right.$ appropriate to the present discussion, calculation of $W_{a b}$ is straightforward if we assume that the partial widths in each channel are described by a Porter-Thomas distribution. If this condition is not satisfied a more advanced treatment is required (e.g. KKM).

Next, we relate the transmission coefficients obtained from an optical model to the energy average of the partial widths appearing in the Breit-Wigner formula. We sum Eq. 2 over outgoing channels $b$ to yield a total reaction cross section,

$$
\sigma_{a}^{J}(E)=\sum_{b} \sigma_{a b}^{J}(E)=\pi \lambda^{2} \omega_{a}^{J} \sum_{\lambda} \frac{\Gamma_{\lambda a} \Gamma_{\lambda}}{\left(E-E_{\lambda}\right)^{2}+\frac{1}{4} \Gamma_{\lambda}^{2}},
$$

in which the total width now appears in the resonance numerators. Taking the energy average of this expression yields

$$
\left\langle\sigma_{a}^{J}(E)\right\rangle=\pi \lambda^{2} \omega_{a}^{J} 2 \pi \frac{\left\langle\Gamma_{a}\right\rangle}{D} .
$$

For simplicity we assume spinless projectile and target, so that the statistical factor $\omega_{a}^{J}$ is just $(2 l+1)$, where $l$ is the orbital angular momentum, and $l$ is sufficient to define the channel $a$. The reaction cross section is then

$$
\left\langle\sigma_{l}(E)\right\rangle=\pi \lambda^{2}(2 l+1) 2 \pi \frac{\left\langle\Gamma_{l}\right\rangle}{D} .
$$

The optical model also yields a value for the energy-averaged reaction cross section, based on absorption in a complex potential instead of an explicit resonance treatment. The optical model value for the reaction cross section for orbital angular momentum $l$ is

$$
\left\langle\sigma_{l}(E)\right\rangle_{\text {optical }}=\pi \lambda^{2}(2 l+1) T_{l},
$$

where $T_{l}$ is the calculated transmission coefficient. Comparison of the above two expressions yields the key result

$$
T_{c}=2 \pi \frac{\left\langle\Gamma_{c}\right\rangle}{D},
$$

where in the general case $c$ is the complete set of angular-momentum quantum numbers that define a channel, not necessarily limited to the orbital angular momentum. This result is valid in the limit $<\Gamma_{c} / D>\ll 1$, and again a more complicated treatment is necessary if this condition is not satisfied. In particular, transmission coefficients must lie between 0 (no absorption) and 1 (complete absorption), whereas there is no upper limit for $\left\langle\Gamma_{c}\right\rangle / D$. A reasonable ansatz that is frequently used to enforce physical limits on $T_{c}$ (but without rigorous justification) is

$$
T_{c}=1-\exp \left(-2 \pi \frac{\left\langle\Gamma_{c}\right\rangle}{D}\right) .
$$

By using Eq. 13 to replace the widths by transmission coefficients in Eq. 8 and summing over total angular momentum $J$, we may express the energy-averaged cross section as

$$
\sigma_{a b}^{\mathrm{HF}}(E)=\sum_{J}\left\langle\sigma_{a b}^{J}(E)\right\rangle=\pi \lambda^{2} \sum_{J} \omega_{a}^{J} \frac{T_{a} T_{b}}{\sum_{c} T_{c}} W_{a b},
$$


which is the Hauser-Feshbach formula with width-fluctuation corrections.

In the interests of simplicity we have ignored several important details of angular momentum coupling and parity conservation. To include these features, we recognize that there is also a sum over the two parities of the compound system, that there are additional sums over angular quantum numbers required to define each channel, and that all sums (except the ones over total angular momentum and parity) are restricted by angular momentum and parity conservation. The complete expression is

$$
\sigma_{a b}^{\mathrm{HF}}(E)=\pi \lambda^{2} \sum_{J \Pi} \omega_{a}^{J} \frac{\sum_{\left\{j_{a}\right\}} T_{a} \sum_{\left\{n_{b}\right\}} \sum_{\left\{j_{b}\right\}} T_{b} W_{a b}}{\sum_{c} \sum_{\left\{n_{c}\right\}} \sum_{\left\{j_{c}\right\}} T_{c}},
$$

where $\Pi$ is the parity of the compound system and $\left\{j_{c}\right\}$ represents the set of angular momenta required to specify the channel. For spinless particles $\left\{j_{c}\right\}$ is $l$, the orbital angular momentum. For spin $1 / 2$ particles $\left\{j_{c}\right\}$ is $l$ plus an additional quantum number, either the channel spin $s$ or $j$, which is obtained by coupling the orbital angular momentum to the particle spin. For photons $\left\{j_{c}\right\}$ represents the multipolarity. The sum on $c$ in the denominator represents the sum over the various 2-body partitions of the compound system for which decay is energetically possible. $\left\{n_{c}\right\}$ is the set of states in the residual nuclei, and the notation $\sum_{\left\{n_{c}\right\}}$ implies a sum over the discrete states and an integral over a continuous spectrum represented by a level density. In the denominator $\sum_{\left\{n_{c}\right\}}$ sums over all final states in the partition $c$ that are energetically reachable. In the numerator $\sum_{\left\{n_{b}\right\}}$ is a sum over whatever final states are intended; this could be as little as a single state or as much as the complete set of energetically reachable states. The factor $W_{a b}$ is inside all sums in the numerator, since it depends on the angular momenta in both channels. The complete expression for the reaction cross section, which is the generalization of Eq. 12, is

$$
\sigma_{a}^{r e a c}(E)=\pi \lambda^{2} \sum_{J \Pi} \omega_{a}^{J} \sum_{\left\{j_{a}\right\}} T_{a} .
$$

Because of the width fluctuation corrections, the above Hauser-Feshbach expressions do not factor into a formation cross section in channel $a$ and a decay probability into channel $b$ (for each value of total angular momentum and parity). When width correlations are negligible $W_{a b}=1$, and then factorization is possible; this is the justification for the compound-nuclear hypothesis (due to Niels Bohr) of independence of formation and decay, which holds separately for each value of total angular momentum and parity in the Hauser-Feshbach theory. While this subject is very complicated, some simple observations are possible:

- Except for elastic scattering, width fluctuation corrections are small when there are many open channels.

- For elastic scattering, channels $a$ and $b$ are the same, and there are strong correlations between incident and exit channels. When many channels are open this leads to an enhancement of the elastic cross section by a factor of 3 in the small $\left\langle\Gamma_{a} / D\right\rangle$ limit considered here, and a factor of 2 in the opposite limit $\left\langle\Gamma_{a} / D\right\rangle \gg 1$. 
- Whereas elastic scattering is enhanced, inelastic and reaction cross sections are depleted by the width fluctuations. The depletion rarely exceeds 10 to $20 \%$, even at fairly low energies (below approximately $2 \mathrm{MeV}$ ). As channels open up with increasing energy the width fluctuation correction quickly becomes unimportant except for elastic scattering.

Often we are interested in reactions leading to excited states of the final nucleus and want to sum over all states of the final nucleus within an energy interval $d E_{b}$ around an excitation energy $E_{b}$. This implies a sum over all final spins $I_{b}$ and both parities $\Pi_{b}$. The final states are characterized by a level density $\rho\left(E_{b}, I_{b}, \Pi_{b}\right)$. In this case we specialize Eq. 16 as

$$
\frac{d \sigma_{a b}^{\mathrm{HF}}}{d E_{b}}=\pi \lambda^{2} \sum_{J \Pi} \omega_{a}^{J} \frac{\sum_{\left\{j_{a}\right\}} T_{a}}{\sum_{c} \sum_{\left\{n_{c}\right\}} \sum_{\left\{j_{c}\right\}} T_{c}} \sum_{I_{b} \Pi_{b}} \sum_{\left\{j_{b}\right\}} T_{b} \rho\left(E_{b}, I_{b}, \Pi_{b}\right),
$$

where we have dropped the width fluctuation correction, which is usually negligible in this case. The transmission coefficients $T_{b}$ are evaluated at an energy for the outgoing particle that leads to an excitation energy $E_{b}$ in the residual nucleus.

A variant of the Hauser-Feshbach formula is useful in the analysis of surrogate reactions and in the study of compound-nuclear reactions in which multiple particles are emitted sequentially from an initial compound nucleus. We temporarily ignore width fluctuation corrections, so that independence of formation and decay applies. Then a generalized HauserFeshbach expression is obtained by writing Eq. 16 as

$$
y_{b}=\sum_{J \Pi} \mathcal{F}_{J \Pi}\left(E_{c m p d}\right) \mathcal{G}_{J \Pi}^{b}\left(E_{c m p d}\right),
$$

where $\mathcal{F}_{J \Pi}\left(E_{c m p d}\right)$ represents the population of compound nucleus levels of spin $J$ and parity $\Pi$ at excitation energy $E_{c m p d}$, and $\mathcal{G}_{J \Pi}^{b}\left(E_{c m p d}\right)$ is the branching ratio of this population into channel $b$; i.e.,

$$
\mathcal{G}_{J \Pi}^{b}=\frac{\sum_{\left\{n_{b}\right\}} \sum_{\left\{j_{b}\right\}} T_{b}}{\sum_{c} \sum_{\left\{n_{c}\right\}} \sum_{\left\{j_{c}\right\}} T_{c}} .
$$

For the 2-step Hauser-Feshbach reaction of Eq. 16, $\mathcal{F}_{J \Pi}$ is the formation cross section given by the incident-channel transmission coefficients, and $y_{b}$ is the cross section for production of channel $b$. However, we may replace this description of $\mathcal{F}_{J \Pi}$ by any other process that yields a well-defined compound nucleus. For a sequential multistep reaction, $\mathcal{F}_{J \Pi}$ is the population produced by earlier stages in the reaction, and $y_{b}$ is the population in channel $b$ produced by the current stage. For a surrogate reaction, the population $\mathcal{F}_{J \Pi}$ characterizes the distribution of highly-excited final states produced in another reaction such as $\left(p, p^{\prime}\right)$ or $\left({ }^{3} H e, \alpha\right)$; these states subsequently decay according to the branching ratio $\mathcal{G}_{J \Pi}^{b}$. Since the transmission coefficients in the numerator of the branching ratios also appear in the denominator, width fluctuation corrections should be incorporated in the above expression. If formation of the compound nucleus represented by $\mathcal{F}_{J \Pi}$ is entirely independent of its decay, width fluctuation corrections can be applied to the branching ratios alone, without invalidating independence of formation and decay. The multistep reactions and surrogate reactions mentioned here are such cases. Otherwise the full expression of Eq. 16 must be used. 
Finally, we note that all of the expressions above have been derived for angle-integrated cross sections. There are corresponding Hauser-Feshbach expressions for differential cross sections, which differ from the above by the inclusion of the appropriate angular momentum coupling coefficients. When a spatial direction is defined, such as by the incident particle in a standard 2-step Hauser-Feshbach reaction, the energy averaging over the underlying resonances implies an angular distribution that is symmetric about 90 degrees with respect to the spatial direction. The same averaging procedure shows that there are no polarization effects. These results may be used to distinguish between direct and compound-nuclear reactions, since direct reactions are asymmetric (usually peaked in the forward hemisphere) and frequently show strong polarization effects.

\section{The Weisskopf-Ewing formula}

In this section we show conditions under which the conservation of total angular momentum and parity in the Hauser-Feshbach expression can be neglected. The cross sections and branching ratios can then be expressed entirely in terms of reaction cross sections, without requiring the explicit use of transmission coefficients. The key approximation that leads to this simplification is that the branching ratios $\mathcal{G}_{J \Pi}^{b}$ in Eq. 20 are independent of $J$ and $\Pi$. The following conditions are sufficient to achieve this:

- The energy of the compound nucleus is sufficiently high that nearly all channels into which it can decay are dominated by integrals over the level density. The fraction of decays proceeding to discrete states must be small.

- Width fluctuations are unimportant. This condition will be satisfied if the previous one is.

- Transmission coefficients are independent of the spin $I_{c}$ of the states reached in channel c. This condition is well satisfied, since the dependence of transmission coefficients on target spin is known to be small in nearly all cases.

- The spin dependence of the level density is proportional to $\left(2 I_{c}+1\right)$ and does not depend on parity. The proportionality to $\left(2 I_{c}+1\right)$ is valid for spins that are not too large, since the level density can be factored as

$$
\rho_{c}\left(E_{c}, I_{c}, \Pi_{c}\right)=\rho_{c}^{\prime}\left(E_{c}\right)\left(2 I_{c}+1\right) \exp \left\{-\frac{\left(I_{c}+\frac{1}{2}\right)^{2}}{2 \sigma_{c}^{2}\left(E_{c}\right)}\right\},
$$

where $\sigma_{c}$ is the spin cutoff parameter for the residual nucleus in channel $c$. Typical values for $\sigma_{c}$ are $4-8$. Although the proportionality to $\left(2 I_{c}+1\right)$ is necessary for a rigorous derivation of the Weisskopf-Ewing reaction model from the Hauser-Feshbach formula, the Weisskopf-Ewing result is still approximately correct at higher spins. This will be commented upon later. As for the parity, statistical-mechanical techniques for 
calculating the parity dependence of level densities indicate that this dependence is very weak at sufficiently high excitation energies that the first condition above is valid.

If the branching ratios $\mathcal{G}_{J \Pi}^{b}$ are indeed independent of $J$ and $\Pi$, as we will show when the above conditions are satisfied, then the Hauser-Feshbach expression Eq. 19 simplifies to the Weisskopf-Ewing form

$$
y_{b}=\mathcal{F}\left(E_{c m p d}\right) \mathcal{G}^{b}\left(E_{c m p d}\right)
$$

where

$$
\mathcal{F}\left(E_{c m p d}\right)=\sum_{J \Pi} \mathcal{F}_{J \Pi}\left(E_{c m p d}\right) .
$$

This expression exhibits independence of formation and decay, unrestricted by conservation of total angular momentum and parity. The integrals in the denominator of $\mathcal{G}^{b}$ extend over the kinematically accessible region of the level spectrum in the residual nucleus in each channel $c$ appearing in the denominator.

The lack of dependence of $\mathcal{G}_{J \Pi}^{b}$ on the parity $\Pi$ of the compound system follows from the assumption that the final-state level densities are independent of parity and the fact that both final-state parities are summed over. We therefore drop the parity index in $\mathcal{G}_{J \Pi}^{b}$.

To show that $\mathcal{G}_{J}^{b}$ is independent of $J$, we write the branching ratio as

$$
\mathcal{G}_{J}^{b}=\frac{\mathcal{J}_{b}}{\sum_{c} \int_{0}^{E_{c}^{m a x}} d E_{c} \mathcal{J}_{c}}
$$

where $\mathcal{J}_{c}$ is a sum over transmission coefficients and level densities for channel $c$; the sum is over all final-state spins $I_{c}$ and over all values of the quantum numbers for the outgoing particle that satisfy angular momentum conservation. For simplicity, we consider only spinless particles with transmission coefficients $T_{l}^{c}$ characterized by orbital angular momentum $l$. The final results do not depend on this restriction. In this case, the quantities $\mathcal{J}_{c}$ are

$$
\mathcal{J}_{c}=\sum_{I_{c}=I_{c}^{\min }}^{\infty} \sum_{l=\left|J-I_{c}\right|}^{J+I_{c}} T_{l}^{c}\left(\epsilon_{c}\right) \rho_{c}\left(E_{c}, I_{c}\right),
$$

where $\epsilon_{c}$ is the kinetic energy of the outgoing particle that leaves an excitation energy $E_{c}$ in the residual nucleus, and $I_{c}^{\text {min }}$ is 0 or $1 / 2$, according to whether the residual nucleus is even or odd mass, respectively. We now assume the level density may be factorized as $\left(2 I_{c}+1\right) \rho_{c}^{\prime}$, and interchange the two sums with the help of the function $\Delta\left(j_{1}, j_{2}, j_{3}\right)$, defined as 1 if the three angular momenta satisfy the vector relationship $\overrightarrow{\mathrm{j} 3}=\overrightarrow{\mathrm{J} 1}+\overrightarrow{\mathrm{J} 2}$ and 0 otherwise. Then

$$
\mathcal{J}_{c}=\rho_{c}^{\prime}\left(E_{c}\right) \sum_{l I_{c}} T_{l}^{c}\left(\epsilon_{c}\right)\left(2 I_{c}+1\right) \Delta\left(J, l, I_{c}\right),
$$

where restrictions on $l$ and $I_{c}$ are provided by the $\Delta$ function. We carry out the sum on $I_{c}$, using the identity

$$
\sum_{I_{c}}\left(2 I_{c}+1\right) \Delta\left(J, l, I_{c}\right)=(2 J+1)(2 l+1),
$$


which is easily shown by counting magnetic substates in the coupled and uncoupled systems represented by $\vec{I}_{c}=\vec{l}+\vec{J}$. Then $\mathcal{J}_{c}$ becomes

$$
\mathcal{J}_{c}=(2 J+1) \rho_{c}^{\prime}\left(E_{c}\right) \sum_{l=0}^{\infty}(2 l+1) T_{l}^{c}\left(\epsilon_{c}\right) .
$$

This completes the demonstration that $\mathcal{G}_{J}^{b}$ is independent of $J$, since each term in both numerator and denominator in Eq. 24 contains the factor $(2 J+1)$, which therefore cancels.

To reach the traditional Weisskopf-Ewing form for $\mathcal{G}^{b}$, we note that (see also Eq. 12) the reaction cross section is given by

$$
\sigma_{c}^{r e a c}=\pi \lambda_{c}^{2} \sum_{l=0}^{\infty}(2 l+1) T_{l}^{c}\left(E_{c}\right)
$$

By using this relation and Eq. 28 we can write the branching ratio as

$$
\mathcal{G}^{b}=\frac{k_{b}^{2} \sigma_{b}^{\text {reac }}\left(\epsilon_{b}\right) \rho_{b}^{\prime}\left(E_{b}\right)}{\sum_{c} \int_{0}^{E_{c}^{\text {max }}} d E_{c} k_{c}^{2} \sigma_{c}^{\text {reac }}\left(\epsilon_{c}\right) \rho_{c}^{\prime}\left(E_{c}\right)},
$$

where the wave number $k_{c}$ is the inverse of the reduced wavelength $\lambda_{c}$ in channel $c$. This is the Weisskopf-Ewing expression for the branching ratio. The quantities $\sigma_{c}^{r e a c}$, often called inverse cross sections, can usually be obtained from optical model calculations or inferred from experiment. The inverse cross section represents the cross section for absorption of the ejected particle as if it were incident on the excited nucleus in channel $c$, and it is assumed that the cross section is the same for absorption on any state in the nucleus in channel $c$. At sufficiently high energies $\sigma_{c}^{\text {reac }}$ is approximately $\pi R^{2}$ (the area of the nucleus) for any ejected particle except for photons. For low-energy neutrons, $\sigma_{c}^{\text {reac }}$ is proportional to $1 / \sqrt{\epsilon_{c}}$. We repeat that the Weisskopf-Ewing expression and its lack of reference to total angular momentum and parity conservation is valid for any type of ejected particle. The derivation for nonzero spin is more tedious but contains the same essential steps that were shown here, in particular the factoring out of $(2 J+1)$ in the expression for $\mathcal{J}_{c}$. For a two-step reaction reaction from channel $a$ to channel $b$, Eq. 17 shows that the sum over transmission coefficients in Eq. 23 for $\mathcal{F}$ is $\sigma_{a}^{r e a c}$, so that the complete expression for the cross section becomes

$$
\frac{d \sigma_{a b}^{\mathrm{WE}}}{d E_{b}}=\sigma_{a}^{\text {reac }} \frac{k_{b}^{2} \sigma_{b}^{\text {reac }}\left(\epsilon_{b}\right) \rho_{b}^{\prime}\left(E_{b}\right)}{\sum_{c} \int_{0}^{E_{c}^{\text {max }}} d E_{c} k_{c}^{2} \sigma_{c}^{\text {reac }}\left(\epsilon_{c}\right) \rho_{c}^{\prime}\left(E_{c}\right)},
$$

which is the Weisskopf-Ewing counterpart to Eq. 18.

As noted earlier, the Weisskopf-Ewing formalism is useful even when the compoundnuclear spin $J$ is comparable to or greater than the spin cutoff parameters $\sigma_{c}$. A qualitative argument shows why this should be so, even though this result cannot be derived rigorously from the Hauser-Feshbach formalism. Starting with compound spin $J$, the distribution of spins $I_{c}$ in each channel $c$ is spread roughly evenly about $J$, since it is given by the various values of the coupling of $J$ to the angular momentum of the outgoing particle. If we replace 
$I_{c}$ by an average value $J$ in the exponential factor in the level density expression of Eq. 21 and assume the spin cutoff factor is approximately the same in all channels, this factor cancels because it appears in all terms in the numerator and denominator of $\mathcal{G}_{J}^{b}$, and the Weisskopf-Ewing result is obtained.

To finish the description of the Weisskopf-Ewing formula, we note that the density $\rho^{\prime}$ is not quite the total level density (i.e., the level density summed over all spins and both parities). The relation between the total level density $\rho^{t o t}$ and $\rho^{\prime}$ is

$$
\rho_{c}^{t o t}\left(E_{c}\right)=4 \sigma_{c}^{2}\left(E_{c}\right) \rho_{c}^{\prime}\left(E_{c}\right) .
$$

The energy dependence of the spin cutoff factor is weak and varies slowly with mass number. Thus it is a reasonable approximation to use $\rho^{\text {tot }}$ rather than $\rho^{\prime}$ in Eqs. 30 and 31 since the factors $4 \sigma^{2}$ are nearly the same in both numerator and denominator.

\section{Application to surrogate reactions}

In this section we apply the results of the previous sections to the analysis of surrogate reactions. We begin with the Hauser-Feshbach formulation. We wish to obtain the compoundnuclear part of a cross section for a reaction from a channel $a$ to a channel $b$, which is given by Eq. 19 in the form

$$
\sigma_{a b}=\sum_{J \Pi} \mathcal{F}_{J \Pi}^{a}\left(E_{c m p d}\right) \mathcal{G}_{J \Pi}^{b}\left(E_{c m p d}\right),
$$

where the target in the incident channel $a$ is in general unstable. However, we can make the highly excited compound nucleus $Y^{*}$ in another reaction $X(x, y) Y^{*}$, with an angle- and energy-differential cross section

$$
\frac{d \sigma_{X Y}}{d \Omega_{y} d E_{c m p d}}\left(\overrightarrow{k_{x}}, \overrightarrow{k_{y}}\right)=\sum_{J \Pi} \frac{d \sigma_{X Y}^{J \Pi}}{d \Omega_{y} d E_{c m p d}}\left(\overrightarrow{k_{x}}, \overrightarrow{k_{y}}\right),
$$

where the left-hand side is the experimentally observable cross section, and the right-hand side is the decomposition of the observable cross section into cross sections leaving the compound nucleus $Y^{*}$ in specific angular momentum and parity values $J$ and $\Pi$. This decomposition must be provided by a reaction model calculation. We assume that the final state of the reaction $X(x, y) Y^{*}$ is a fully equilibrated compound nucleus. This assumption requires careful examination, which is beyond the scope of this tutorial. We define the probability that $X(x, y) Y^{*}$ forms $Y^{*}$ with the quantum numbers $J \Pi$ as

$$
\mathcal{F}_{J \Pi}^{\text {surr }}=\frac{d \sigma_{X Y}^{J \Pi}}{d \Omega_{y} d E_{c m p d}} / \sum_{J^{\prime} \Pi^{\prime}} \frac{d \sigma_{X Y}^{J^{\prime} \Pi^{\prime}}}{d \Omega_{y} d E_{c m p d}} .
$$

In the surrogate technique, the outgoing particle $y$ is measured in coincidence with the same outgoing particle that appears as the outgoing particle in channel $b$ in the desired reaction of Eq. 33. The experimentally observable probability of this coincidence (appropriately 
corrected for solid angle and efficiency) is

$$
P_{b}^{\text {surr }}=\sum_{J \Pi} \mathcal{F}_{J \Pi}^{\text {surr }}\left(E_{c m p d}\right) \mathcal{G}_{J \Pi}^{b}\left(E_{c m p d}\right) .
$$

The particle in channel $b$ observed in coincidence with $y$ may decay to either specific final states or a distribution of states; the desired reaction Eq. 33 must be interpreted accordingly. A specific example relevant to experiments currently under way is the observation of gammas following neutron emission from the compound nucleus, observed in coincidence with $\alpha$ particles in reactions of the type $X\left({ }^{3} \mathrm{He}, \alpha\right) Y$. In this case $b$ represents neutron emission to all energetically available final states, and the gammas are a measure of the cross section for emission of these neutrons when properly interpreted with a model for the gamma cascades leading to the observed gammas. Another example is the observation of fission fragments in coincidence with the outgoing particle in a reaction that populates the fissioning nucleus. In this case also, $b$ is a distribution of final states, represented by the transition states built on the saddle-point configuration of the fissioning system.

Eqs. 33 and 36 are the Hauser-Feshbach formulation of the surrogate reaction problem. These expressions contain the same branching ratios $\mathcal{G}_{J \Pi}^{b}$, but they are weighted differently because the $J \Pi$ distributions are different in the two reactions. In fact, the difference in these distributions may be very great. An extreme case is the use of the surrogate technique to determine a low-energy $(n, \gamma)$ reaction, since the neutron will deposit very little angular momentum in the compound nucleus compared with that typically brought in by the $X(x, y) Y^{*}$ reaction. The main theoretical challenge in interpreting a surrogate reaction measurement is to determine the angular-momentum decomposition probabilities $\mathcal{F}_{J \Pi}^{\text {surr }}$ and branching ratios $\mathcal{G}_{J \Pi}^{b}$ in Eq. 36 accurately. If these are well determined, the branching ratios can be inserted in Eq. 33 to yield the desired cross section. Note that the coincidence probabilities $P_{b}^{\text {surr }}$ are the only experimental results available to help in this determination. It is particularly desirable for experiments to be carried out over a wide range of angles in the $X(x, y) Y^{*}$ reaction, since the probabilities $\mathcal{F}_{J \Pi}^{\text {surr }}$ are angle-dependent, and obtaining the same $\sigma_{a b}$ for the various angles provides an important consistency check on the procedure.

If the branching ratios $\mathcal{G}_{J \Pi}^{b}$ are independent of $J$ and $\Pi$, we are in the Weisskopf-Ewing limit and the analysis simplifies significantly. The conditions that allow this simplification were discussed in the previous section. In particular, it is important that the particles in channel $b$ populate a wide distribution of final states. From Eqs. 17 and 35 the sums over the $\mathcal{F}_{J \Pi}$ factors are

$$
\sum_{J \Pi} \mathcal{F}_{J \Pi}^{a}=\sigma_{a}^{r e a c}
$$

and

$$
\sum_{J \Pi} \mathcal{F}_{J \Pi}^{\text {surr }}=1
$$

Then Eqs. 33 and 36 simplify to

$$
\sigma_{a b}=\sigma_{a}^{r e a c} \mathfrak{G}^{b}
$$

and

$$
P_{b}^{s u r r}=\mathcal{G}^{b}
$$


Comparison of the last two expressions yields the simple result

$$
\sigma_{a b}=\sigma_{a}^{r e a c} P_{b}^{s u r r}
$$

which is entirely independent of the branching ratios. The desired cross section is expressed as the product of an experimental quantity, the measured coincidence probability, and a reaction cross section that can be calculated with an optical model to a few percent accuracy.

The Weisskopf-Ewing result provides a useful prescription for preliminary analysis of a surrogate experiment, which may be quite accurate when the conditions for its validity are satisfied. The full Hauser-Feshbach analysis appears to be highly model dependent, but this model dependence may be weak if the conditions for validity of the Weisskopf-Ewing formalism are even approximately satisfied, since most of the model dependence drops out in the latter approach. This tentatively positive conclusion needs to be tested for each surrogate reaction by appropriate sensitivity studies, such as by varying the $J \Pi$ distribution of the population parameters in Eq. 36.

It should be pointed out that the surrogate reaction technique considered above can only determine the equilibrium portion of the desired reaction, since the assumption of a long-lived, fully equilibrated compound nucleus is fundamental to the analysis. Therefore any necessary preequilibrium component must be calculated separately and added to the cross section resulting from the surrogate reaction analysis. For reactions with neutrons up to 10 or $20 \mathrm{MeV}$ the preequilibrium component is no larger than about $20 \%$, and thus determining the preequilibrium component with standard techniques tuned to systematics in nearby nuclei may not contribute excessively to the overall error.

Analyzing a surrogate reaction by Eq. 36 requires a suitably modified Hauser-Feshbach code. Modifying STAPRE, a code routinely used for reaction calculations at Livermore, will not be difficult. This code has an array that contains the compound-nuclear cross sections $\mathcal{F}_{J \Pi}^{a}$ produced by the incident particle. These values can be replaced by the probabilities $\mathcal{F}_{J \Pi}^{\text {surr }}$, and the transmission coefficients for the incident particle can be set to zero so that they play no role in the Hauser-Feshbach denominators. The modified code should then be fully adequate for the surrogate reaction analysis, including the possibility of including width-fluctuation corrections and the ability to calculate gamma cascades when necessary. Similar modifications should be possible with other codes.

\section{Acknowledgments}

This work was performed under the auspices of the U.S. Department of Energy by the University of California, Lawrence Livermore National Laboratory under contract No. W7405-Eng-48. 